Vietnam Journal of Mechanics, NCST of Vietnam Vol. 25, 2003, No $2(170-185)$

\title{
NUMERICAL SOLUTION FOR CONSISTENT INITIAL CONDITIONS OF CONSTRAINED MECHANICAL SYSTEMS
}

\author{
Dinh VAN Phong \\ Department of Applied Mechanics, Hanoi University of Technology
}

\begin{abstract}
The article deals with the problem of consistent initial values of the system of equations of motion which has the form of the system of differential-algebraic equations. Direct treating the equations of mechanical systems with particular properties enables to study the system of DAE in a more flexible approach. Algorithms and examples are shown in order to illustrate the considered technique.
\end{abstract}

\section{Introduction}

The system of equations of motion of a mechanical system leads to the problem of solving the system of ordinary differential equations (ODE) or the mixed system of differential-algebraic equations (DAE). If the set of independent generalised coordinates is used, the system of equations of motion has the form of ordinary differential equation. In contrast, if the set of dependent generalised coordinates is used, the system of equations of motion will have the form of differential-algebraic equations. Due to the generality and complexity of these systems of equations numerical integration is usually applied in order to get the solution. Various schemes for ordinary differential equations and differential-algebraic equations can be used for this purpose.

Here in this article we will focus our attention on the problem of the initial values of the system of differential-algebraic equations, since unlike the ordinary differential equations initial conditions for differential-algebraic equations can not be chosen arbitrarily: they have to meet the constraints equation and their derivatives. This is one major difference between differential-algebraic equations and ordinary differential equations. It can be seen as one source of difficulties in numerically solving differential-algebraic equations.

The problem of consistency of initial conditions is a central part for all types of differential-algebraic equations which must be addressed as part of the problem solution, see e.g. [2], [9], [18], [19], [21] etc. In recent years many mathematical researchers were interested in the general problem of consistency of initial condition for first order ordinary differential equations which can be described in general by equation:

$$
\mathbf{f}\left(\mathbf{y}, \mathbf{y}^{\prime}, x\right)=\mathbf{0}
$$


where $x$ is the independent variable, $\mathbf{y}$ respectively $\mathbf{y}^{\prime}$ are the vector of dependent variables respectively their derivations, $\mathbf{f}$ is the vector of functions to be integrated. The general definition of consistency and various classification with algorithms could be given, see e.g. [1], [8], [15], [20] etc. However due to the generality of the problem these algorithms can not be so simple and convenient. Therefore we will release these general algorithms and invoke the specialised case of mechanical systems. The structure of equations of motion of mechanical system allows us to deal the problem with more simple and convenient algorithm.

For constrained mechanical system the conventional method is using Lagrange multipliers. This approach leads to the system of differential-algebraic equations with the presence of "algebraic" quantities in the form of multipliers. The alternative approach is the principle of compatibility which is applicable even for more general cases of the the systems with nonideal constraints. Both forms of equations of motion will be discussed below. Further the extended case for the constrained mechanical system with so-called nongeneralised coordinates will be treated.

\section{Initial conditions for various forms of the equations of motion}

\subsection{The system of equations of motion with the principle of compatibility}

\subsubsection{Basic case}

Firstly, consider the differential-algebraic equations of motion in the form:

$$
\begin{aligned}
\mathbf{A} \ddot{\mathbf{q}} & =\mathbf{h}+\mathbf{r}, \\
\mathbf{g}(\mathbf{q}) & =\mathbf{0}, \\
\mathbf{D}^{T} \mathbf{r} & =\mathbf{0},
\end{aligned}
$$

which is constructed according to the principle of compatibility, see [3], [4], [5], [14]. This is the case of constrained holonomic scleronomous system with ideal constraints. The extension for more general cases will be done in further discussion. Recall that the system is described by an $n$-dimensional vector of generalised coordinates $\mathbf{q}$ and is constrained by an $s$-dimensional vector of constraints $\mathbf{g}$ according to (3), $\mathbf{A}$ is an $(n \times n)$-dimensional matrix of inertia, $\mathbf{h}$ is the vector, absorbing Coriolis, centrifugal and generalised forces and $\mathbf{r}$ is vector of reaction forces and $\mathbf{D}$ is an $(n \times(n-s))-$ dimensional matrix, which is derived from the condition of ideality of the constraints. The matrices $\mathbf{A}, \mathbf{D}$ and $\mathbf{h}$ are functions of generalised coordinate $\mathbf{q}$ and generalised velocity $\dot{\mathbf{q}}$.

When speaking about initial conditions of the system (2.1), (2.2) and (2.3) the following considerations should be clear. From the point of view of motion of mechanical system, the system has $(n-s)$ degrees of freedom. In order to avoid the physically meaningless cases $(2(n-s))$ initial conditions should be available: $(n-s)$ conditions for positions and $(n-s)$ conditions for velocities. They are independent and, of course, not contradict to each other. In the other hand, from the point of 
view of numerical integration, the numerical scheme requires the initial values of all quantities, i.e.

$$
\mathbf{q}_{0}=\mathbf{q}(0), \quad \dot{\mathbf{q}}_{0}=\dot{\mathbf{q}}(0), \quad \ddot{\mathbf{q}}_{0}=\ddot{\mathbf{q}}(0) \text { and } \quad \mathbf{r}_{0}=\mathbf{r}(0) .
$$

In other words we should have $\mathrm{n}$ initial conditions for the components of vector of generalised coordinates q, $n$ initial conditions for the components of the vector of generalised velocities $\dot{\mathbf{q}}, n$ initial conditions for the components of the vector of generalised accelerations $\ddot{\mathbf{q}}, n$ initial conditions for the components of the vector of generalised reaction forces $r$. These values should be consistent: they should meet the constraint equations and their derivatives.

Therefore for assuring the consistency of initial conditions, the constraint equation (2.2) is differentiated twice. One gets:

$$
\mathbf{G}(\mathbf{q}) \dot{\mathbf{q}}=\mathbf{0}
$$

and

$$
\mathbf{G} \ddot{\mathbf{q}}+\mathbf{g}^{(0)}=0,
$$

where $\mathbf{G}$ is an $(s \times n)$-dimensional Jacobian matrix of the vector $\mathbf{g}$ with respect to the vector $\mathbf{q}$ and $\mathbf{g}^{(0)}$ is an $s$-dimensional vector.

The definition for the consistency of initial conditions of the system (2.1), (2.2) and (2.3) is as follows:

Definition D.1. The initial values (2.4) of the system (2.1), (2.2) and (2.3) are consistent if they satisfy following:

$$
\begin{aligned}
& \mathbf{A}\left(\mathbf{q}_{0}, \dot{\mathbf{q}}_{0}, t_{0}\right) \ddot{\mathbf{q}}_{0}=\mathbf{h}\left(\mathbf{q}_{0}, \dot{\mathbf{q}}_{0}, t_{0}\right)+\mathbf{r}_{0}, \\
& \mathbf{g}\left(\mathbf{q}_{0}\right)=\mathbf{0}, \\
& \mathbf{G}\left(\mathbf{q}_{0}\right) \dot{\mathbf{q}}_{0}=\mathbf{0}, \\
& \mathbf{G}\left(\mathbf{q}_{0}\right) \ddot{\mathbf{q}}_{0}+\mathbf{g}^{(0)}=\mathbf{0}, \\
& \mathbf{D}^{T}\left(\mathbf{q}_{0}, \dot{\mathbf{q}}_{0}\right) \mathbf{r}_{0}=\mathbf{0} .
\end{aligned}
$$

The system (2.7), (2.8), (2.9), (2.10) and (2.11) in this definition does not completely determine the initial values. Logically, some values of positions and velocities may be chosen, corresponding to the number of degrees of freedom of the system. In the case of consideration this number, as discussed above, is $(n-s)$. The rest of positions, velocities and other quantities should be determined, in order to satisfy the Definition D.1.

A sequential initialisation process can be provided in the 3 phases. First the position problem must be solved, then the velocity analysis must be performed and subsequently an acceleration analysis yields all accelerations together with reaction 
forces. The algorithm for defining consistent initial conditions for the system (2.1), (2.2) and (2.3) can be written as follows:

\section{Algorithm A.1}

1. Set $n_{p}=n-s$.

2. Set $n_{v}=n_{p}$.

3. Choose $n_{p}$ independent initial conditions for positions.

4. Choose $n_{v}$ independent initial conditions for velocities.

5. Determine the rest of initial conditions for positions from the equation (2.8).

6. Determine the rest of initial conditions for velocities from the equation (2.9).

7. Determine the rest of initial conditions for acceleration and reaction forces from the equation (2.7), (2.10) and (2.11)

In this algorithm the number $n_{p}$ and $n_{v}$ are defined explicitly according to our problem (2.1), (2.2) and (2.3). They are corresponding to $(n-s)$ degrees of freedom of the system under consideration and in the extension of the algorithm for more general cases, as shown below, these values will be modified for specific cases. $n_{p}$ values of positions and $n_{v}$ values of velocities respectively may be chosen at the time instant $t=t_{0}$, or in other words at the beginning of numerical integration these values should be supplied explicitly. Clearly, these initial values of coordinates should be independent and enable defining the rest of all quantities. For checking the independency of coordinates the partioning approach with SVD or LU etc. could be applied e.g. [10], [18], [21] etc.

In the step 5 of above algorithm the complete set of initial values for position is defined. The matrix equation (2.8) presents the system of $s$, in general nonlinear, equations for $s$ unknowns, i.e. the rest of initial values of generalised coordinates. Similarly, in the step 6 we should solve the system of $s$ equations for $s$ unknowns. For the system described in (2.1), (2.2) and (2.3), the velocity analysis leads to the problem of solving the linear system of algebraic equations.

In the last step, the complete set of initial values is determined. In this step we have the system of $(2 n)$ algebraic equations for $(2 n)$ unknowns. This system has the following form:

$$
\left[\begin{array}{c:cc}
\mathbf{A} & -\mathbf{I} \\
\hdashline \mathbf{G} & - & -- \\
\hdashline \mathbf{0} & - & - \\
\hdashline \mathbf{D}^{T}
\end{array}\right]\left[\begin{array}{c}
\ddot{\mathbf{q}}_{0} \\
\hdashline \mathbf{r}_{0}
\end{array}\right]=\left[\begin{array}{c}
\mathbf{h} \\
\hdashline-\mathbf{g}_{0} \\
\hdashline \mathbf{0}
\end{array}\right]
$$

2.1.2. Extension for more general cases

Now consider the case of the system which is restricted by the constraint equation in the more general form:

$$
\mathbf{g}(\mathbf{q}, \dot{\mathbf{q}})=\mathbf{0}
$$


Assume that the vector function $\mathbf{g}$ includes both types of constraints: holonomic and nonholonomic. It means that we have $s_{p}$ equations in which only generalised coordinates $q_{i}, i=1,2, \ldots, n$, appear, and $s_{v}$ equation, in which generalised velocities $\dot{\mathrm{q}}_{i}, i=1,2, \ldots, n$, appear. Clearly, the total number of constraints is $s$ :

$$
s_{p}+s_{v}=s
$$

Formally we can separate (2.13) into two groups:

$$
\begin{array}{r}
\mathbf{g}_{v}(\mathbf{q}, \dot{\mathbf{q}})=\mathbf{0} \\
\mathbf{g}_{p}(\mathbf{q})=\mathbf{0}
\end{array}
$$

where $g_{v}$ and $g_{p}$ are vectors of dimension of $\left(s_{v} \times n\right)$ and $\left(s_{p} \times n\right)$ respectively. The Jacobian matrices are assumed to be well posed i.e. Jacobian $\mathbf{G}_{p}$ of the vector $\mathbf{g}_{p}$ with respect to $\mathbf{q}$ and Jacobian $\mathbf{G}_{v}$ of the vector $\mathbf{g}_{v}$ with respect to $\dot{\mathbf{q}}$, are both full rank.

When some nonholonomic constraint is imposed on the system, the generalised velocities are restricted, but not the generalised coordinates. It means that on the position level one more coordinate could be chosen freely, or it should be supplied at the beginning of integration process. Therefore the number of coordinates on the position level is:

$$
n_{p}=n-s_{p} .
$$

The number of initial values $n_{v}$ for velocities is the same as $s$. Hence, the Algorithm A.1 is easily extended for the case under consideration. It is only necessary to check at the beginning the number of $s_{p}$ : how many constraint equations include explicitly generalised velocities.

For acceleration analysis the same techniques as in the previous case can be applied. For this purpose the constraint equation (2.13) is transformed into the form:

$$
\mathrm{G} \ddot{\mathrm{q}}+\mathrm{g}^{(0)}=\mathbf{0} .
$$

Remind that this equation looks like the equation (2.6), but the matrix $\mathbf{G}$ is constructed in different way. Since the vector function $\mathbf{g}$ includes both types of equations (in the first one the generalised velocities appear explicitly and in the second one only generalised coordinates appear), the vector functions $\mathbf{g}_{i}, i=1,2, \ldots, s$, should be derivated once or two times in order to get generalised accelerations in the equations.

The algorithm for determining the consistent initial values for this case is as follows:

\section{Algorithm A.2}

1. 1.a Determine the number $s_{p}, s_{v}$. 
2. Set $\quad n_{v}=n-s$.

1.b Set $n_{p}=n-s_{p}$.

3. Choose $n_{p}$ initial conditions for positions.

4. Choose $n_{v}$ initial conditions for velocities.

5. Determine the rest of initial conditions for positions from the equation (2.16).

6. Determine the rest of initial conditions for velocities from the equation (2.13).

7. Determine the initial values for accelerations and reaction forces from the equations (2.7), (2.11) and (2.18).

Note that in this case the velocity analysis in step 5 does not perform the linear system of algebraic equations but it leads to the problem of solving the system of nonlinear equations due to the general form of equations (2.13). In the acceleration analysis with the modified structure of the matrix $\mathrm{G}$ we get again the problem of solving the linear system of algebraic equations in the form of (2.12).

It is possible to extend the algorithm for more general case when the constraint equations may include some restriction on acceleration level. The constraint equations have the form:

$$
\mathbf{g}(\mathbf{q}, \dot{\mathbf{q}}, \ddot{\mathbf{q}})=0 .
$$

Again with regard to the highest derivatives of the vector $\mathbf{q}$ in each equation we can separate the equations (2.19) into three groups:

$$
\begin{array}{r}
\mathbf{g}_{a}(\mathbf{q}, \dot{\mathbf{q}}, \ddot{\mathbf{q}})=\mathbf{0} \\
\mathbf{g}_{v}(\mathbf{q}, \dot{\mathbf{q}})=\mathbf{0} \\
\mathbf{g}_{p}(\mathbf{q})=0 .
\end{array}
$$

The dimensions of vectors $\mathbf{g}_{a}, \mathbf{g}_{v}$ and $\mathbf{g}_{p}$ correspond to the numbers $s_{a}, s_{v}$ and $s_{p}$ of the constraints in which accelerations, velocities or only generalised coordinates appear:

$$
s=s_{a}+s_{v}+s_{p} .
$$

Obviously we assume again that these constraints are well posed, i.e. the Jacobian matrices of corresponding vectors with respect to $\ddot{\mathbf{q}}$, $\dot{\mathbf{q}}$ or $\mathbf{q}$ are full rank.

The similar consideration, as in the previous case, can be applied. Each of the constraint equations on the acceleration level $(2.20)$ presents two integration constants. It means that we can choose arbitrarily two initial conditions: one for the position level and one for the velocity level. Hence the number for initial values of position is:

$$
n_{p}=n-s_{p} .
$$

The number for initial values for velocities is:

$$
n_{v}=n-s_{v}-s_{p} .
$$


Clearly the position analysis can be provided with the matrix equation (2.22). For velocity and acceleration analysis the constraint equations (2.21), (2.22) should be converted to the derivated forms. From (2.21) one gets:

$$
\mathbf{G}_{v} \ddot{\mathbf{q}}+\mathbf{g}_{v}^{(0)}=\mathbf{0},
$$

where $\mathbf{G}_{v}$ is an $\left(s_{v} \times n\right)$-dimensional Jacobian matrix of the vector $\mathbf{g}_{v}$ with respect to $\dot{\mathbf{q}}$ and $\mathbf{g}_{v}^{(0)}$ is an $s_{v}$-dimensional vector.

Derivating the equation (2.22) once and two times yields:

$$
\mathbf{G}_{p} \dot{\mathbf{q}}=\mathbf{0}
$$

and

$$
\mathbf{G}_{p} \ddot{\mathbf{q}}+\mathbf{g}_{v}^{(0)}=\mathbf{0},
$$

where $\mathbf{G}_{p}$ is an $\left(s_{p} \times n\right)$-dimensional Jacobian matrix of the vector $\mathbf{g}_{p}$ with respect to $\mathrm{q}$ and $\mathbf{g}_{v}^{(0)}$ is an $s_{p}$-dimensional vector.

The consequent steps of algorithm for this case can be described as following.

Algorithm A.3

1. 1.a From the constraint equations determine the corresponding numbers $s_{p}$, $s_{v}, s_{a}$.

1.b Set $n_{p}=n-s_{p}$.

2. Set $n_{v}=n-s_{p}-s_{v}$.

3. Choose $n_{p}$ initial conditions for positions.

4. Choose $n_{v}$ initial conditions for velocities.

5. Determine the rest of initial conditions for positions from the equation (2.22), i.e.

$$
\mathbf{g}_{p}\left(\mathbf{q}_{0}\right)=\mathbf{0} .
$$

6. Determine the rest of initial conditions for velocities from the equation (2.21) and $(2.27)$, i.e.

$$
\begin{aligned}
& \mathbf{g}_{v}\left(\mathbf{q}_{0}, \dot{\mathbf{q}}_{0}\right)=\mathbf{0}, \\
& \mathbf{G}_{p} \dot{\mathbf{q}}_{0}=\mathbf{0} .
\end{aligned}
$$

7. Determine the initial values for accelerations and reaction forces from the equations $(2.7),(2.11),(2.20),(2.26)$ and (2.28), i.e.

$$
\begin{aligned}
& \mathbf{A}\left(\mathbf{q}_{0}, \dot{\mathbf{q}}_{0}, t_{0}\right) \ddot{\mathbf{q}}_{0}=\mathbf{h}\left(\mathbf{q}_{0}, \dot{\mathbf{q}}_{0}, t_{0}\right)+\dot{\mathbf{r}}_{0} \\
& \mathbf{D}^{T} \mathbf{r}_{0}=\mathbf{0} \\
& \mathbf{g}_{a}\left(\mathbf{q}_{0}, \dot{\mathbf{q}}_{0}, \ddot{\mathbf{q}}_{0}\right)=\mathbf{0} \\
& \mathbf{G}_{v} \ddot{\mathbf{q}}_{0}+\mathbf{g}_{v}^{(0)}=\mathbf{0} \\
& \mathbf{G}_{p} \ddot{\mathbf{q}}_{0}+\mathbf{g}_{p}^{(0)}=\mathbf{0}
\end{aligned}
$$


Note that in this algorithm the calculation provided in the steps $5,6,7$ for positions, velocities and accelerations analysis presents in general the problem of solving the system of nonlinear equations. In the step 5 the system consists of $s_{p}$ equations for the rest of $s_{p}$ values of generalised coordinates. In the step 6 the system has $\left(s_{p}+s_{v}\right)$ equations for $\left(s_{p}+s_{v}\right)$ values of velocities, yet unknown. Finally in the step 7, the system in total presents $(2 n)$ equations of $2 n$ components of $\ddot{q}_{0}$ and $\mathbf{r}_{0}$.

Obviously the Algorithms A.1 and A.2 can be included in the more general Algorithm A.3. In these particular cases we have $s_{a}=0$ and $s_{v}=0$ respectively. However due to the specific form of the obtained equations the technique for solution of the linear system of algebraic equations could be applied instead of the system of nonlinear equations.

So far we discuss only scleronomous system, i.e. time variable, namely $t$, does not appear explicitly in the constraint equations. The extension of above algorithms for the rheonomous system can be provided easily. The appearance of the time variable in the equations of constraints does not affect the flowchart of consecutive steps in the algorithms. Only we should take into account this time variable $t$ when derivating the constraint equations. Hence some forms of equations may be changed due to the partial derivative of the functions with respect to time. Namely, the equation (2.27) now has the form:

$$
\mathbf{G}_{p} \dot{\mathbf{q}}+\mathbf{g}_{p}^{t}=\mathbf{0},
$$

where $\mathbf{g}_{p}^{t}$ is a vector of partial derivative of $\mathbf{g}_{p}$ with respect to the time variable $t$. Consequently the form of (2.31) in Algorithm A.3 is changed in similar way. The rest of the algorithm is unchanged formally.

In order to reduce the number of equation to be integrated, sometimes the reduced form of equation of motion is used, see [14]. In this case the reaction forces are excluded from the system of equation. The above algorithms are still valid with corresponding modification in the acceleration analysis (e.g. step 7), since there is no need of initial values for reaction forces.

For the system with nonideal constraints we can also apply this approach. Instead of the condition of ideality (2.33) in Algorithm A.3, we dispose other $(n-s)$ conditions:

$$
\begin{aligned}
\mathbf{f}\left(\mathbf{r}_{0}\right) & =\mathbf{0}, \\
\mathbf{D}_{1}^{T} \mathbf{r}_{0} & =\mathbf{0} .
\end{aligned}
$$

Recall here that $\mathbf{f}$ is a vector function, supplied by specific requirements of problem and $D_{1}$ is a matrix which could be generated by the technique similar to the technique for the matrix $\mathbf{D}$ in (2.3). The rest of the Algorithm A.3 is valid for the general case with nonideal constraints. 


\subsection{The system of equations of motion with Lagrange multipliers}

For ideal constraints the conventional method is using Lagrange multipliers. We can apply easily above approach for this form of equations of motion. Instead of reaction forces we will have the Lagrange multipliers $\lambda=\left[\lambda_{j}\right], j=1,2, \ldots, s$, where $s$ is the number of constraints. The system of differential-algebraic equations in this case with holonomic scleronomous constraints is as follows:

$$
\begin{aligned}
& \mathbf{A} \ddot{\mathbf{q}}=\mathbf{h}+\mathbf{G}^{T} \lambda, \\
& \mathbf{g}(\mathbf{q})=\mathbf{0}
\end{aligned}
$$

The meaning of quantities is the same as in the system of (2.1), (2.2) and (2.3).

The definition in the Section 2.1 and Algorithm A.1 can be easily extended for the system (2.40) and (2.41). The only difference is the fact that the dimension of the vector $\lambda$ is only $s$. Hence we can state the definition for consistent initial values of $\mathbf{q}_{0}, \dot{\mathbf{q}}_{0}, \ddot{q}_{0}, \lambda$ for the system (2.40) and (2.41) if the following holds

$$
\begin{aligned}
& \mathbf{A}\left(\mathbf{q}_{0}, \dot{\mathbf{q}}_{0}, t_{0}\right) \ddot{\mathbf{q}}=\mathbf{h}\left(\mathbf{q}_{0}, \dot{\mathbf{q}}_{0}, t_{0}\right)+\mathbf{G}^{T} \lambda, \\
& \mathbf{g}\left(\mathbf{q}_{0}\right)=\mathbf{0}, \\
& \mathbf{G}\left(\mathbf{q}_{0}\right) \dot{\mathbf{q}}_{0}=\mathbf{0}, \\
& \mathbf{G}\left(\mathbf{q}_{0}\right) \ddot{\mathbf{q}}_{0}+\mathbf{g}^{(0)}=\mathbf{0} .
\end{aligned}
$$

and the Algorithm A.1 is applied for this system. Clearly, the system for defining $\lambda_{0}$ and $\ddot{q}_{0}$ in the step 7 is (2.42) and (2.45). The rest of the algorithm remains unchanged.

Similarly, for the extended cases: the system with nonholonomic constraints (2.13) or constraints with acceleration (2.19), we can use the Algorithms A.2 and A.3 with minor modification in the acceleration analysis in the step 7. The extension for the rheonomous system as discussed in the previous section is valid. It is worth reminding that for the system with ideal constraints both approaches: the principle of compatibility and using Lagrange multipliers, are applicable but for nonideal constraint the only way is the principle of compatibility.

\subsection{The mechanical system with nongeneralised coordinates}

In engineering application there are cases when in the system of equations of motion appear so-called nongeneralised coordinates. The term "nongeneralised" is due the fact that no inertia forces are associated with them. Hence the nongeneralised coordinates do not increase the number of differential equations of motion. Example of such mechanical system can be found in the contact problem of multibody dynamics or in the control problem.

Detail discussion about deriving equation of motion for such system is out of scope of this article, see e.g. [6] but recall here that we can use the principle of compatibility. The reaction forces of the constraints that correspond to these 
coordinates are zero. Consider the holonomic system with $n$ generalised coordinates described by the vector $\mathbf{q}$ and $m$ nongeneralised coordinates described by a vector p.

If the number of constraints $s$ is equal to the number of nongeneralised coordinates $m$, i.e $s=m$, the system of equations of motion is in the form of differertialalgebraic equation as follows:

$$
\begin{aligned}
& \mathbf{A}(\mathbf{q}, \mathbf{p}) \ddot{\mathbf{q}}=\mathbf{h}(\mathbf{q}, \mathbf{p}, t) \\
& \mathbf{g}(\mathbf{q}, \mathbf{p}, t)=\mathbf{0}
\end{aligned}
$$

Keep in mind that $\mathbf{A}$ is of $(n \times n)$-dimension, $\mathbf{q}$ and $\mathbf{h}$ are of $(n \times 1)$-dimension, $\mathrm{p}$ is of $m$-dimension and $\mathbf{g}$ is of $s$-dimension (in this case $m$-dimension). The system of consideration has $n$ degrees of freedom, hence the initial values for $\mathbf{q}$ and $\dot{\mathbf{q}}$ are known before integrating process. The initial values for nongeneralised coordinates can be defined in the similar way according to the Algorithm A.1, but in this case the evaluation of "algebraic" quantity $p_{0}$ can be performed directly if $p$ appear only in the equation (2.47), separately from the evaluation of $\ddot{q}_{0}$.

For the case when the number of constraints $s$ is greater than the number of nongeneralised generates $m$, i.e. $s>m$, the system of constraints is split into two groups. The first group is used to construct differential equation according to the principle of compatibility and the reaction forces, corresponding to the second group of constraints are zero. The equation of motion in the form of the system of differential-algebraic equations is obtained and the basic Algorithm A.1 for evaluation of consistent initial conditions can be applied even for this case. It is worth emphasising that this technique with the principle of compatibility is also valid for nonholonomic system, see e.g.[6].

\section{Illustrated examples}

For illustration we will consider two examples. The first one is simple case of spatial four bar mechanism with dependent set of coordinates and the second one is the case of the system with nonholonomic constraints and nongeneralised coordinate.

\subsection{Spatial four bar mechanism}

Consider a spatial four-bar mechanism shown in figure 1. In order to illustrate the constraint condition, a two-degree-of-freedom mechanical system in the form of the double pendulum is used. Each of the uniform bar 1 and 2 is 2 units long with its centroid located at the center. The constraint is presented by a body with unit length and neglible mass that connects the end of body 2 and point $(0,2,0)$. Two generalised coordinates are chosen: $q_{1}$ is the orientation of the bar 1 in respect to $x$-axis and $q_{2}$ is the orientation of bar 2 in respect to $z$-axis, see [10]. 
From the distance condition of point $[0,2,0]$ and end point of bar 2 we can write the constraint condition:

$$
8-8 \cos q_{1} \sin q_{2}-4 \sin q_{1}=0 .
$$

This is the case of holonomic constraint (2.2) with

$$
n=2, \quad s=1 \quad \text { and } \quad n_{p}=n_{v}=1 .
$$

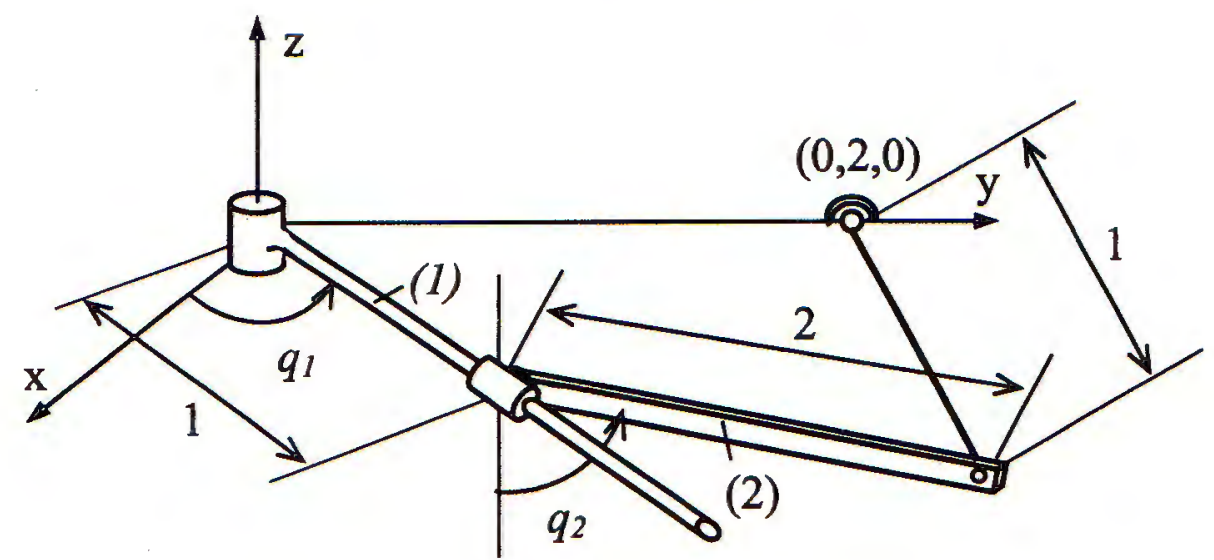

Fig. 1. Spatial four-bar mechanism

With Lagrange multipliers one gets the equation of motions as follows:

$$
\begin{aligned}
& {\left[\begin{array}{ccc}
\frac{4 m_{1}}{3}+m_{2}\left(1+\frac{4 \sin ^{2} q_{2}}{3}\right) & m_{2} \cos q_{2} & 8 \sin q_{1} \sin q_{2}-4 \cos q_{1} \\
m_{2} \cos q_{2} & \frac{4 m_{2}}{3} & -8 \cos q_{1} \cos q_{2} \\
8 \sin q_{1} \sin q_{2}-4 \cos q_{1} & -8 \cos q_{1} \cos q_{2} & 0
\end{array}\right]\left[\begin{array}{c}
\ddot{q}_{1} \\
\ddot{q}_{2} \\
\lambda
\end{array}\right]} \\
& =\left[\begin{array}{c}
m_{2} \sin q_{2}\left(\dot{q}_{2}^{2}-\frac{8 \dot{q}_{1} \dot{q}_{2} \cos q_{2}}{3}\right) \\
-m_{2} g \sin q_{2}+\frac{4 m_{2} \dot{q}_{1} \cos q_{2} \sin q_{2}}{3} \\
-\left(8 \cos q_{1} \sin q_{2}+4 \sin q_{1}\right) \dot{q}_{1}^{2}-16 \sin q_{1} \cos q_{2} \dot{q}_{1} \dot{q}_{2}-8 \cos q_{1} \sin q_{2} \dot{q}_{2}
\end{array}\right] .
\end{aligned}
$$

Since the DOF of the system is 1 , the initial condition is e.g.:

$$
q_{1}(0)=q_{1}^{0}, \quad \dot{q}_{1}(0)=\dot{q}_{1}^{0} .
$$

In order to get the solution of the system the set of initial conditions should be determined.

Following the Algorithm A1 and the discussion in the Section 2.2 the position analysis is provided: $\dot{q}_{2}(0)$ is determined from the equation (3.1). This performs the solving nonlinear equation. The velocity $\dot{q}_{2}(0)$ is determined from the equation:

$$
\left(8 \sin q_{1} \sin q_{2}-4 \cos q_{1}\right) \dot{q}_{1}-8 \cos q_{1} \cos q_{2} \dot{q}_{2}=0 \text {. }
$$


Finally $\ddot{q}_{1}(0), \ddot{q}_{2}(0)$ and $\lambda(0)$ are determined from the system, consisting of equation (3.3) and following equation:

$$
\begin{aligned}
& \left(8 \sin q_{1} \sin q_{2}-4 \cos q_{1}\right) \ddot{q}_{1}-8 \cos q_{1} \cos q_{2} \ddot{q}_{2}= \\
& =-\left[\left(8 \cos q_{1} \sin q_{2}+4 \sin q_{1}\right) q_{1}^{2}+16 \sin q_{1} \cos q_{2} \dot{q}_{1} \dot{q}_{2}+8 \cos q_{1} \sin q_{2} \dot{q}_{2}\right] .
\end{aligned}
$$

Obviously, the solution of problem (3.1), (3.5) or (3.6) can be provided only with numerical methods for nonlinear system of equation and for linear system of algebraic equations. With the complete set of the values of $q_{1}, q_{2}, \dot{q}_{1}, \dot{q}_{2}, \ddot{q}_{1}, \ddot{q}_{2}, \lambda$ for the time instance $t=0$, the numerical integration can be started.

It is worth noting that it is possible to define independent generalised coordinate that leads to nonlinear differential equation of motion and the problem of initial conditions disappears. But in this case the form of equation is strongly nonlinear and very complex. This is the advantage of the concept with dependent coordinates, however, some problems such as consistency of initial condition should be solved.

\subsection{Vehicle model in manoeuvre}

Consider the motion of a simple model of an automobile in a horizontal plane, Figure 2, see [6], [7], and [12]. The body of the car is considered as a rigid body and its configuration is determined by the coordinates $x, y$ and $\varphi$, i.e. the coordinates of the mass center and the angle between the body's lengthwise axe and $x$-axis of the fixed frame. The controlling component also is represented by a body, the position of which is determined by angle $\theta$, i.e. the angle of its lengthwise axe with respect to the car body. The car body has a mass $m_{0}$, the moment of inertia about the vertical axis passing through the mass center is $I_{0}$. The controlling component has a moment of inertia $I$ about the vertical axis passing through its center but its mass is small in comparison with the mass of the car and, therefore, it could be neglected.

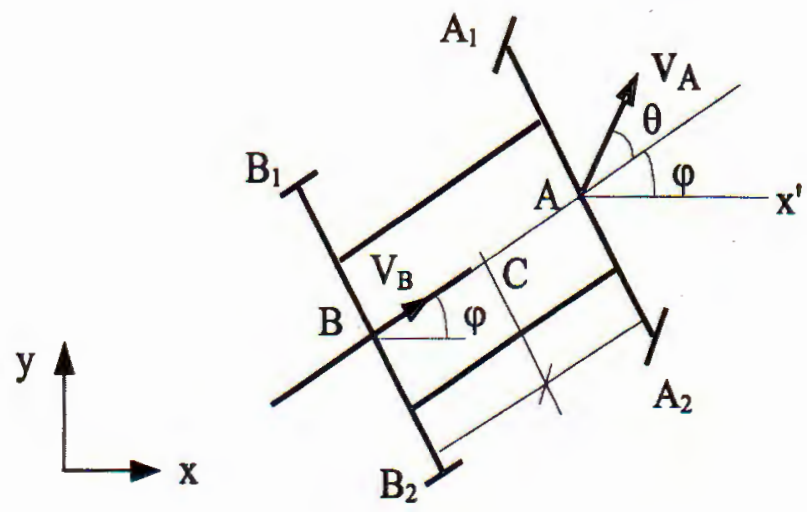

Fig. 2. Planar vehicle model in manoeuvre

Now, consider the motion of the car with the assumption that the planes of front wheels are parallel with the lengthwise axe of the controlling component, which 
is affected by controlling moment $M_{d}$. When changing direction, the system has following nonholonomic constraints:

$$
\begin{aligned}
& -\dot{x} \sin \varphi+\dot{y} \cos \varphi+b \varphi=0, \\
& -\dot{x} \sin (\varphi+\theta)+\dot{y} \cos (\varphi+\theta)+a \cos \theta \dot{\varphi}=0,
\end{aligned}
$$

where $b$ and $a$ are distances from the mass center to the front and rear axe respectively. The car body has a total length $L, L=a+b$.

Assume that the car is controlled to follow the trajectory of the mass center, which has the form:

$$
x^{2}+(y-R)^{2}-R^{2}=0 .
$$

Using the principle of compatibility in the reduction form we can write the differential equation of motion in the form, see [6]:

$$
\begin{aligned}
& m_{0}(L \cos \theta \cos \varphi-b \sin \theta \sin \varphi) \ddot{x}+m_{0}(L \cos \theta \sin \varphi+b \sin \theta \cos \varphi) \ddot{y} \\
& +\left(I_{0}+I\right) \sin \theta \ddot{\varphi}+I \sin \theta \ddot{\theta}=\left[F_{0}-k_{1}\left(\dot{x}^{2}+\dot{y}^{2}\right)\right] L \cos \theta-k_{2} \sin \theta \dot{\varphi}^{2}, \\
& I \ddot{\varphi}+I \ddot{\theta}-M_{d}=0 .
\end{aligned}
$$

This is the case of nonholonomic system due to the presence of constraints (3.7) and (3.8). The nongeneralised coordinate in our case is the controlling moment $M_{d}$. Hence the system of equation of motion that consists of five equations (3.7), (3.8), (3.9), (3.10) and (3.11), is a mixed system of differential-algebraic equation for five unknowns $x, y, \varphi, \theta$ and $M_{d}$. For this constrained mechanical system the following holds:

$$
n=4, \quad s=3, \quad s_{p}=1, \quad s_{v}=2, \quad n_{p}=3, \quad n_{v}=1, \quad m=1 .
$$

The initial conditions for this problem can be chosen e.g. as follows:

$$
x(0)=x_{0}, \quad \varphi(0)=\varphi_{0}, \quad \theta(0)=\theta_{0}, \quad \dot{x}(0)=\dot{x}_{0} .
$$

Keep in mind the set of initial conditions can not be chosen arbitrary due to the full rank condition of Jacobian matrices for defining independent set of coordinates. For example, we can not choose the set including both coordinates $x$ and $y$ in this case.

The rest of values of consistent initial conditions is determined from the position, velocity and acceleration analysis. Firstly the value of $y(0)$ is evaluated from equation (3.9). The values of $\dot{y}(0), \dot{\varphi}(0), \dot{\theta}(0)$ are determined from the system of equations that includes (3.7), (3.8) and the following equation:

$$
x \dot{x}+(y-R) \dot{y}=0 .
$$


The accelerations $\ddot{x}(0), \ddot{y}(0), \ddot{\varphi}(0), \ddot{\theta}(0)$ and the algebraic quantity $M_{d}(0)$ is evaluated from the system of five equations that consists of (3.10), (3.11) and three following equations:

$$
\begin{aligned}
& \dot{x}^{2}+x \ddot{x}+\dot{y}^{2}+(y-R) \ddot{y}=0, \\
& -\sin \varphi \ddot{x}-(\dot{x} \cos \varphi+\dot{y} \sin \varphi) \dot{\varphi} \cos \varphi \ddot{y}+b \ddot{\varphi}=0, \\
& -\sin (\varphi+\theta) \ddot{x}-[\dot{x} \cos (\varphi+\theta)+\dot{y} \sin (\varphi+\theta)] \dot{\varphi} \\
& -[\dot{x} \cos (\varphi+\theta)+\dot{y} \sin (\varphi+\theta)+a \sin \theta \dot{\varphi}] \dot{\theta} \\
& +\cos (\varphi+\theta)+a \cos \theta \ddot{\varphi}=0 .
\end{aligned}
$$

Again with the complete set of values of $x, y, \varphi, \theta, \dot{x}, \dot{y}, \dot{\varphi}, \dot{\theta}, \ddot{x}, \ddot{y}, \ddot{\varphi}, \ddot{\theta}$ and $M_{d}$ for the time instance $t=0$, the numerical integration can be started.

In this example we can see that with the appearance of nongeneralised quantities, e.g. controlling forces etc., the problem of consistency of initial condition is inevitable. The procedure of numerical integration required even the initial values of these quantities but they are in general unknown at the beginning. The example illustrates a generally applicable numerical method for searching these values for numerical integration.

\section{Conclusion}

In this article we are interested in numerical determining consistent initial conditions for the system of equations of motion of the mechanical system in the form of differential algebraic equations. Two problems should be emphasized when dealing with this particular system.

The first problem is the appearance of the "algebraic" quantities in this system. They may be Lagrange's multipliers (when using Langrange equations with multipliers) or generalised reaction forces (when using principle of compatibility) or nongeneralised coordinates. The initial values of these quantities are unknowns before the integration process and should be determined. Normally, as shown in this article, they can be evaluated together with generalised accelerations in the acceleration analysis after position and velocity analysis.

The second problem is the type of constraint equations. According to these equations the appropriate set of initial values of independent coordinates and velocities can be chosen. The rest of consistent initial values is determined by performing position an velocity analysis.

Our approach with considered algorithms can be easily converted to the computer modules and included in the packages for analysis and simulation of constrained mechanical system. Clearly this required some other numerical modules, such as solution of the system of nonlinear equation or coordinates partioning etc.

This publication is completed with financial support from the National Basic Research Program in Natural Sciences. 


\section{REFERENCES}

1. Brown P. N., Hindmarsh A. C., Petzold L. R. Consistent initial condition calculation for differential-algebraic systems. SIAM J. Sci. Comput., 19, pp.1495-1512, 1998.

2. Dinh Van Phong. Differential-algebraic equations and study of motion of mechanical system. Proceedings of the fifth national conference on mechanics, Hanoi, Vol.1, pp.121-125, 1993.

3. Dinh Van Phong. Principle of compatibility and criteria of ideality in study of constrained mechanical systems. Strojnicky casopis (Journal of Czech and Slovak mechanical engineering), 47, N. 1, pp. 2-11, Bratislava, 1996.

4. Do Sanh. On the Motion of Constrained Mechanical Systems. The thesis of doctor of science, Hanoi University of Technology, Hanoi, 1984, (in Vietnamese).

5. Do Sanh, Dinh Van Phong. The principle of compatibility and computational mechanics. Proceedings of the NCST of Vietnam, Vol.7, No. 1, pp. 3-8, 1995.

6. Do Sanh, Dinh Van Phong, Nguyen Nhat Le, Du Quoc Thinh. Dynamics of the vehicles in changing direction. Proceedings of International colloquium in mechanics of solids, fluids structures and interactions, Nhatrang, pp. 36-43, 2000.

7. Dobronravov V. V. Bases of Nonholonomic Mechanics, Vyska Skola, Moscow, 1976, (in Russian).

8. Fuhrer C., Lemkuler B. J. Numerical solution of differential-algebraic equation for constrained mechanical system. Numer. Math. 59, pp. 55-69, 1991.

9. Garcia de Jalon, Bayo E. Kinematic and dynamic simulation of multibody systems: The real-time challenge. Springer, NewYork/Berlin, 1996.

10. Haug E. J. Intermediate Dynamics. Prentice Hall, Englewood Cliffs, 1992

11. Kane T. R., Levison D. A. Dynamics: theory and application. McGraw Hill, 1985.

12. Lobas L. G. Nonholonomic Models of a Wheeled Vehicle. Kiev Naukova Dumka, 1986, (in Russian).

13. Nejmark J. I., Fufajew N. A. Dynamics of Nonholonomic System. Nauka, Moscow, 1984, (in Russian).

14. Nguyen Nhat Le, Dinh Van Phong, Do Sanh. On numerical methods for constrained mechanical systems. Proceedings of the IUTAM Symposium on recent development in non-linear oscillation of mechanical system (NOMES), Kluwer Academic Publisher, pp. 207-216, 2000.

15. Rabier P. J., Rheiboldt. Nonholonomic motion of rigid mechanical systems from a DAE viewpoint. SIAM, 2000.

16. Schiehlen W. Multibody System Handbook, Springer-Verlag, Berlin, 1990.

17. Schiehlen W. Multibody system dynamics: Roots and perspectives. Multibody system dynamics 1: 149-188, Kluwer Academic Publishers, 1997. 
18. Schwerin R. V. Multibody system simulation. Springer, Berlin/Heidelberg, 1999.

19. Shabana A. A. Computational Dynamics. John Willey \& sons, New York, 2001.

20. Soellner E. E., Fuhrer C. Numerical methods in multibody dynamics. Teubner Stuttgart, 1998.

21. Stejskal V., Valasek M. Kinematics and dynamics of machinery. Marcel Dekker, New York, 1996.

Received August 20, 2002

\section{XÁC ĐINHH BẰNG PHƯONG PHÁP SỐ CÁC ĐIỀU KIỆN ĐẦU TƯƠNG THÍCH CƯA CÁC HỆ CƠ HỌC CHỊU LIÊN KẾT}

Bài báo trình bày phương án xác định các điều kiện đầu tương thích cho các hệ phương trình chuyển động có dạng vi phân đại sổ. Việc xét trực tiếp các tính chất của các phương trình chuyển động của các hệ cơ học, cho phép tiếp cận các phương trình vi phân đại số một cách thuận lợi hơn. Các thuật giải và ví dụ được đưa ra để minh họa cho phương pháp được trình bày.

Dinh Van Phong

Department of Applied Mechanics, Hanoi University of Technology 1st Dai Co Viet, Hanoi, Vietnam

Tel: (84) (4) 8680469 Fax: (84) (4) $8683280 \quad$ Home: (84) (4) 8536298

Email: phong@mail.hut.edu.vn 